\title{
Some applications of the Lorentzian holonomy algebras
}

\author{
Anton S. Galaev
}

September 25, 2018

\begin{abstract}
It is shown how one can apply the classification of the holonomy algebras of Lorentzian manifolds to solve some problems. In particular, a new proof to the classification of Lorentzian manifolds with recurrent curvature tensor is given; the classification of twosymmetric Lorentzian manifolds is explained; conformally recurrent Lorentzian manifolds are classified; recurrent symmetric bilinear forms on Lorentzian manifolds are described.
\end{abstract}

\section{Introduction}

While the classification of the Riemannian holonomy algebras is a classical result that has many applications both to geometry and physics, see e.g. [4, 15], the classification of the Lorentzian holonomy algebras has been achieved only recently [10, 17]. We review it in Section 3. The holonomy algebra of a pseudo-Riemannian manifold is an important invariant of the Levi-Civita connection. It provides information about parallel and recurrent tensors on the manifold. Using that property, we solve some problems in Lorentzian geometry. As the first illustration, in Section 6 we give a new and modern proof to the classification of Lorentzian manifolds $(M, g)$ with recurrent curvature tensor $R$, i.e. satisfying

$$
\nabla_{X} R=\theta(X) R
$$

for all vector fields $X$ and a 1-form $\theta$. Originally this classification is achieved in [24]. In Section 7 we discuss Lorentzian symmetric spaces. As a new result, in Section 9 we obtain a classification of Lorentzian manifolds with recurrent conformal Weyl tensor $W$. This generalizes result from $[8,9]$ that gives classification of Lorentzian manifolds with parallel $W$. In Section 10 we explain the result from [2] about the classification of two-symmetric Lorentzian manifolds $(M, g)$, i.e. manifolds satisfying the condition

$$
\nabla^{2} R=0, \quad \nabla R \neq 0
$$

In Section 11 we study recurrent symmetric bilinear forms on Lorentzian manifolds.

\section{Holonomy algebras; parallel and recurrent tensor fields}

Let $(M, g)$ be a connected pseudo-Riemannian manifold of signature $(r, s)$. The holonomy group $G_{x}$ of $(M, g)$ at a point $x \in M$ is the Lie group that consists of the pseudo-orthogonal 
transformations given by the parallel transports along all piecewise smooth loops at the point $x$. It can be identified with a Lie subgroup of the pseudo-orthogonal Lie group $\mathrm{O}(r, s)=$ $\mathrm{O}\left(T_{x} M, g_{x}\right)$. The corresponding subalgebra $\mathfrak{g}_{x}$ of $\mathfrak{s o}(r, s)=\mathfrak{s o}\left(T_{x} M, g_{x}\right)$ is called the holonomy algebra of $(M, g)$ at the point $x \in M$.

The Ambrose-Singer Theorems states that the holonomy algebra $\mathfrak{g}_{x}$ is spanned by the following endomorphisms of $T_{x} M$ :

$$
\tau_{\gamma}^{-1} \circ R_{y}\left(\tau_{\gamma} X, \tau_{\gamma} Y\right) \circ \tau_{\gamma},
$$

where $\gamma$ is a piecewise smooth curve starting at the point $x$ with an end-point $y \in M$, and $X, Y \in T_{x} M$.

Since the manifold $M$ is connected, the holonomy groups (holonomy algebras) of $(M, g)$ at different points are isomorphic, and one may speak about the holonomy group $G \subset \mathrm{O}(r, s)$ (the holonomy algebra $\mathfrak{g} \subset \mathfrak{s o}(r, s))$ of $(M, g)$.

Recall that a tensor field $T$ on $(M, g)$ is parallel if $\nabla T=0$, where $\nabla$ is the Levi-Civita connection; $T$ is called recurrent if $\nabla T=\theta \otimes T$ for a 1 -form $\theta$.

The fundamental principle [4] states that there exists a one-to-one correspondents between parallel tensor fields $T$ on $M$ and tensors $T_{0}$ of the same type at $x$ preserved by the holonomy group (more precisely, by its tensor extension of its representation). Similarly, there exists a one-to-one correspondents between rank one parallel subbundles of a tensor bundle over $M$ and 1-dimensional subspaces of the space of tensors of the same type at $x$ preserved by the holonomy group. Any section of a rank one parallel subbundle of a tensor bundle is a recurrent tensor field. Conversely, any non-vanishing recurrent tensor field defines such parallel subbundle.

If the manifold $M$ is simply connected, then the holonomy group is connected and it is uniquely defined by the holonomy algebra. Then parallel and recurrent tensors may be described in terms of the holonomy algebra.

\section{Classification of the Lorentzian holonomy algebras}

Here we review results from $[17,10]$. Let $(M, g)$ be a simply connected Lorentzian manifold of dimension $n+2, n \geq 0$. Fix a point $x \in M$. The tangent space $\left(T_{x} M, g_{x}\right)$ can be identified with the Minkowski space $\left(\mathbb{R}^{1, n+1}, \eta\right)$. Then the holonomy algebra $(M, g)$ at the point $x$ is identified with a subalgebra $\mathfrak{g} \subset \mathfrak{s o}(1, n+1)$.

We may assume that the holonomy algebra $\mathfrak{g} \subset \mathfrak{s o}(1, n+1)$ of $(M, g)$ is weakly irreducible, i.e. it does not preserve any non-degenerate proper vector subspace of the tangent space. Indeed, if $\mathfrak{g} \subset \mathfrak{s o}(1, n+1)$ is not weakly irreducible, then by the Wu Theorem, $(M, g)$ is at least locally can be decomposed into a product of a Lorentzian manifold and a Riemannian manifold, see e.g. [10]. Thus we assume that $(M, g)$ is locally indecomposable. If $\mathfrak{g} \subset \mathfrak{s o}(1, n+1)$ is irreducible, then $\mathfrak{g}=\mathfrak{s o}(1, n+1)$. Suppose that $\mathfrak{g} \subset \mathfrak{s o}(1, n+1)$ is not irreducible, then $\mathfrak{g}$ preserves an isotropic line in $\mathbb{R}^{1, n+1}$.

The Lie algebra $\mathfrak{s o}(1, n+1)$ can be identified with the space of bivectors $\Lambda^{2} \mathbb{R}^{1, n+1}$ in such a way that

$$
(X \wedge Y) Z=\eta(X, Z) Y-\eta(Y, Z) X .
$$

Let $p \in \mathbb{R}^{1, n+1}$ be an isotropic vector. Fix an isotropic vector $q \in \mathbb{R}^{1, n+1}$ such that $\eta(p, q)=1$. Let $E$ be the orthogonal complement to $\mathbb{R} p \oplus \mathbb{R} q$, then $E \simeq \mathbb{R}^{n}$ is an Euclidean space and we 
get

$$
\mathbb{R}^{1, n+1}=\mathbb{R} p \oplus E \oplus \mathbb{R} q .
$$

Denote by $\mathfrak{s i m}(n)$ the maximal subalgebra in $\mathfrak{s o}(1, n+1)$ preserving the isotropic line $\mathbb{R} p$, then it holds

$$
\mathfrak{s i m}(n)=\mathbb{R} p \wedge q+\mathfrak{s o}(n)+p \wedge E,
$$

here $\mathfrak{s o}(n)=\mathfrak{s o}(E) \simeq \wedge^{2} E$. Any weakly irreducible not irreducible subalgebra $\mathfrak{g} \subset \mathfrak{s o}(1, n+1)$ preserves an isotropic line in $\mathbb{R}^{1, n+1}$, hence $\mathfrak{g}$ is conjugated to a subalgebra of $\mathfrak{s i m}(n)$. The weakly irreducible Lorentzian holonomy algebras $\mathfrak{g} \subset \mathfrak{s i m}(n)$ are the following:

(type I) $\mathfrak{g}=\mathbb{R} p \wedge q+\mathfrak{h}+p \wedge E ; \quad($ type II) $\mathfrak{g}=\mathfrak{h}+p \wedge E ;$

(type III) $\mathfrak{g}=\{\varphi(A) p \wedge q+A \mid A \in \mathfrak{h}\}+p \wedge E$;

(type IV) $\mathfrak{g}=\{A+p \wedge \psi(A) \mid A \in \mathfrak{h}\}+p \wedge E_{1}$,

where $\mathfrak{h} \subset \mathfrak{s o}(n)$ is a Riemannian holonomy algebra, $\varphi: \mathfrak{h} \rightarrow \mathbb{R}$ is a linear map that is zero on the commutant $[\mathfrak{h}, \mathfrak{h}]$; for the last algebra, $E=E_{1} \oplus E_{2}$ is an orthogonal decomposition, $\mathfrak{h}$ annihilates $E_{2}$, i.e. $\mathfrak{h} \subset \mathfrak{s o}\left(E_{1}\right)$, and $\psi: \mathfrak{h} \rightarrow E_{2}$ is a surjective linear map that is zero on $[\mathfrak{h}, \mathfrak{h}]$.

\section{The spaces of curvature tensors}

We will need the following algebraic results. Let $(W, \eta)$ be a pseudo-Euclidean space and $\mathfrak{f} \subset \mathfrak{s o}(W)$ be a subalgebra. The vector space

$$
\mathcal{R}(\mathfrak{f})=\left\{R \in \Lambda^{2} W^{*} \otimes \mathfrak{f} \mid R(X, Y) Z+R(Y, Z) X+R(Z, X) Y=0, X, Y, Z \in W\right\}
$$

is called the space of algebraic curvature tensors of type $\mathfrak{f}$. The space $\mathcal{R}(\mathfrak{f})$ is an $\mathfrak{f}$-module with the action

$$
(\xi \cdot R)(X, Y)=[\xi, R(X, Y)]-R(\xi X, Y)-R(X, \xi Y), \quad \xi \in \mathfrak{f}, R \in \mathcal{R}(\mathfrak{f}) .
$$

From the Ambrose-Singer Theorem it follows that if $\mathfrak{f} \subset \mathfrak{s o}(W)$ is the holonomy algebra of a pseudo-Riemannian manifold $(N, h)$, then the values of the curvature tensor of $(N, h)$ belong to $\mathcal{R}(\mathfrak{f})$ and

$$
\mathfrak{f}=\operatorname{span}\{R(X, Y) \mid R \in \mathcal{R}(\mathfrak{f}), X, Y \in W\},
$$

i.e. $\mathfrak{f}$ is spanned by the images of the elements $R \in \mathcal{R}(\mathfrak{f})$.

The spaces $\mathcal{R}(\mathfrak{g})$ for Lorentzian holonomy algebras $\mathfrak{g} \subset \mathfrak{s i m}(n)$ are found in [11, 12]. Let e.g. $\mathfrak{g}=\mathbb{R} p \wedge q+\mathfrak{h}+p \wedge E$. For the subalgebra $\mathfrak{h} \subset \mathfrak{s o}(n)$ define the space

$$
\mathcal{P}(\mathfrak{h})=\left\{P \in E^{*} \otimes \mathfrak{h} \mid \eta(P(X) Y, Z)+\eta(P(Y) Z, X)+\eta(P(Z) X, Y)=0, X, Y, Z \in E\right\} .
$$

Any $R \in \mathcal{R}(\mathfrak{g})$ is uniquely given by

$$
\lambda \in \mathbb{R}, \vec{v} \in E, P \in \mathcal{P}(\mathfrak{h}), R_{0} \in \mathcal{R}(\mathfrak{h}), \text { and } T \in \operatorname{End}(E) \text { with } T^{*}=T
$$

in the following way:

$$
\begin{aligned}
R(p, q) & =-\lambda p \wedge q-p \wedge \vec{v}, \quad R(X, Y)=R_{0}(X, Y)-p \wedge(P(Y) X-P(X) Y), \\
R(X, q) & =-g(\vec{v}, X) p \wedge q+P(X)-p \wedge T(X), \quad R(p, X)=0
\end{aligned}
$$

for all $X, Y \in E$. For the algebras $\mathfrak{g}$ of the other types, any $R \in \mathcal{R}(\mathfrak{g})$ can be given in the same way and by the condition that $R$ takes values in $\mathfrak{g}$. For example, $R \in \mathcal{R}(\mathfrak{h}+p \wedge E)$ if and only if $\lambda=0$ and $\vec{v}=0$. 


\section{$5 \quad$ Walker metrics and pp-waves}

Consider the local form of a Lorentzian manifold $(M, g)$ with the holonomy algebra $\mathfrak{g} \subset \mathfrak{s i m}(n)$. Since $\mathfrak{g}$ preserves an isotropic line of the tangent space, $(M, g)$ locally admits a parallel distribution of isotropic lines. Locally there exist the so called Walker coordinates $v, x^{1}, \ldots, x^{n}, u$ and the metric $g$ has the form

$$
g=2 \mathrm{~d} v \mathrm{~d} u+h+2 A \mathrm{~d} u+H(\mathrm{~d} u)^{2},
$$

where $h=h_{i j}\left(x^{1}, \ldots, x^{n}, u\right) \mathrm{d} x^{i} \mathrm{~d} x^{j}$ is an $u$-dependent family of Riemannian metrics, $A=$ $A_{i}\left(x^{1}, \ldots, x^{n}, u\right) \mathrm{d} x^{i}$ is an $u$-dependent family of one-forms, and $H$ is a local function on $M$, see e.g. [10]. The vector field $\partial_{v}$ defines the parallel distribution of isotropic lines.

Consider the fields of frames

$$
p=\partial_{v}, \quad X_{i}=\partial_{i}-A_{i} \partial_{v}, \quad q=\partial_{u}-\frac{1}{2} H \partial_{v}
$$

and the distribution $E=\operatorname{span}\left\{X_{1}, \ldots, X_{n}\right\}$. At each point $m$ of the coordinate neighborhood we get the decomposition

$$
T_{m} M=\mathbb{R} p_{m} \oplus E_{m} \oplus \mathbb{R} q_{m},
$$

hence the value $R_{m}$ of the curvature tensor can be expressed in terms of some $\lambda_{m}, \vec{v}_{m}, R_{0 m}, R_{m}$ and $T_{m}$ as above. The space $E_{m}$ is isomorphic to the tangent space of a Riemannian manifolds with a metric from the family $h$, then $R_{0}$ is defined by the curvature tensor of the family of the Riemannian metrics $h$.

It is known [10] that the holonomy algebra of the manifold $(M, g)$ is contained in $p \wedge E \subset \mathfrak{s i m}(n)$ if and only if the metric can be locally written in the form

$$
g=2 \mathrm{~d} v \mathrm{~d} u+\sum_{i=1}^{n}\left(\mathrm{~d} x^{i}\right)^{2}+H(\mathrm{~d} u)^{2}, \quad \partial_{v} H=0 .
$$

Such spaces are called pp-waves.

\section{Lorentzian manifolds with recurrent curvature tensor}

In this section we consider Lorentzian manifolds $(M, g)$ with recurrent curvature tensor $R$, i.e. satisfying (1). Note that for Riemannian manifolds (1), implies $\theta=0$, i.e. the manifold is locally symmetric [16].

Many facts about recurrent spaces, or more generally about $r$-recurrent spaces, and a long list of literature on this topic can be found in the fundamental review of Kaigorodov [16]. There is a recent review by Senovilla [20], where similar problems are considered.

In this section we give a new proof to the following theorem proven by Walker in [24].

Theorem 1 Let $(M, g)$ be a Lorentzian manifold of dimension $n+2 \geq 3$. Then $(M, g)$ is recurrent and not locally symmetric if and only if in a neighborhood of each point of $M$ there exist coordinates $v, x^{1}, \ldots, x^{n}, u$ such that one of the following holds:

I. there exists a function $H\left(x^{1}, u\right)$ such that

$$
g=2 d v d u+\sum_{i=1}^{n}\left(d x^{i}\right)^{2}+H\left(x^{1}, u\right)(d u)^{2} .
$$


II. There exist real numbers $\lambda_{1}, \ldots, \lambda_{n}$ with $\left|\lambda_{1}\right| \geq \cdots \geq\left|\lambda_{n}\right|, \lambda_{2} \neq 0$, and a function $F: U \subset \mathbb{R} \rightarrow \mathbb{R}$ such that

$$
g=2 d v d u+\sum_{i=1}^{n}\left(d x^{i}\right)^{2}+F(u) \lambda_{i}\left(x^{i}\right)^{2}(d u)^{2} .
$$

Moreover, for some system of coordinates $\partial_{1}^{2} H$ is not constant or $\frac{d F}{d u} \neq 0$.

The form of the metric may change from one system coordinates to another, i.g. it can be flat for some systems of coordinates. Examples of such spaces can be constructed taking the metrics of the form (6) with $F(u)=0$ if $|u| \geq \epsilon$ for some $\epsilon>0$, any such metric is flat on the spaces $\left\{\left(v, x^{1}, \ldots x^{n}, u\right)|| u \mid \geq \epsilon\right\}$, hence me may glue these metrics on such flat spaces. In this example the function $F(u)$ is not analytic. Theorem 2 below states that if the manifold $(M, g)$ is analytic, then the metric is the same for all systems of coordinates.

Note that the local metric (6) is symmetric if and only $F$ is a constant, i.e. $\frac{\mathrm{d} F(u)}{\mathrm{d} u}=0$. In this case we get the so called Cahen-Wallach space [6]. Next, the local metric (6) is two-symmetric, i.e. $\nabla^{2} R=0$, if and only if $\frac{\mathrm{d}^{2} F(u)}{(\mathrm{d} u)^{2}}=0$, see Section 10 below. Finally, it is conformally flat if and only if $\lambda_{1}=\cdots=\lambda_{n}[13]$.

\subsection{Proof of Theorem 1}

First we reduce the problem to the case when $(M, g)$ is locally indecomposable.

Lemma 1 Let $(M, g)$ be a recurrent and not locally symmetric Lorentzian manifold. Suppose that $(M, g)$ is locally decomposable, i.e. each point of $M$ has an open neighborhood $U$ such that $\left(U,\left.g\right|_{U}\right)$ is isometric to the product of a Lorentzian manifold $\left(M_{1}, g_{1}\right)$ and a Riemannian manifold $\left(M_{2}, g_{2}\right)$. If $\left.\nabla R\right|_{U} \neq 0$, then $\left(M_{1}, g_{1}\right)$ is recurrent and $\left(M_{2}, g_{2}\right)$ is flat. If $\left.\nabla R\right|_{U}=0$, then both $\left(M_{1}, g_{1}\right)$ and $\left(M_{2}, g_{2}\right)$ are locally symmetric.

Proof. Since $\left(U,\left.g\right|_{U}\right)=\left(M_{1} \times M_{2}, g_{1}+g_{2}\right)$, for the corresponding curvature tensors and their covariant derivatives it holds

$$
\left.R\right|_{U}=R_{1}+R_{2},\left.\quad \nabla R\right|_{U}=\nabla R_{1}+\nabla R_{2}
$$

Suppose that $\left.\nabla R\right|_{U} \neq 0$. Restricting the equality $\nabla R=\theta \otimes R$ to $\left(M_{2}, g_{2}\right)$, we get $\nabla R_{2}=$ $\left.\theta\right|_{M_{2}} \otimes R_{2}$. Since $\left(M_{2}, g_{2}\right)$ is a Riemannian manifold, $\left.\theta\right|_{M_{2}}=0$. Let $X_{1} \in \Gamma\left(T M_{1}\right)$ and $X_{2}, Y_{2} \in$ $\Gamma\left(T M_{2}\right)$, then

$$
\begin{aligned}
0=\nabla_{X_{1}} R_{1}\left(X_{2}, Y_{2}\right)+\nabla_{X_{1}} R_{2}\left(X_{2}, Y_{2}\right) & \\
& =\theta\left(X_{1}\right) R_{1}\left(X_{2}, Y_{2}\right)+\theta\left(X_{1}\right) R_{2}\left(X_{2}, Y_{2}\right)=\theta\left(X_{1}\right) R_{2}\left(X_{2}, Y_{2}\right) .
\end{aligned}
$$

Since $\left.\theta\right|_{U} \neq 0, R_{2}=0$. This proves the lemma.

The condition (1) implies that for any point $m \in M$, the holonomy algebra $\mathfrak{g}_{m}$ of $(M, g)$ preserves the line $\mathbb{R} R_{m} \subset \mathcal{R}\left(\mathfrak{g}_{m}\right)$ in the space of possible values of the curvature tensor at the point $m$.

The only possible irreducible holonomy algebra of $(M, g)$ is the Lorentzian Lie algebra $\mathfrak{s o}(1, n+$ 1) [10]. Form the results of [1] it follows that the only line preserved by $\mathfrak{s o}(1, n+1)$ in the space 
$\mathcal{R}(\mathfrak{s o}(1, n+1))$ consists of curvature tensors defined by the scalar curvature. Consequently the manifold is Einstein and locally symmetric. Hence the holonomy algebra of $(M, g)$ is weakly irreducible and not irreducible and it is contained in $\mathfrak{s i m}(n)$.

The condition that the holonomy algebra $\mathfrak{g}_{m}$ at the point $m \in M$ preserves the line $\mathbb{R} R_{m} \subset$ $\mathcal{R}\left(\mathfrak{g}_{m}\right)$ can be expressed as

$$
\xi \cdot R_{m}=\mu(\xi) R_{m}, \quad \xi \in \mathfrak{g}_{m}
$$

where $\mu: \mathfrak{g}_{m} \rightarrow \mathbb{R}$ is a linear map. Let e.g. $\mathfrak{g}_{m}=\mathbb{R} p_{m} \wedge q_{m}+\mathfrak{h}+p_{m} \wedge E_{m}$. As the $\mathfrak{h}$-module, the space $\mathcal{R}\left(\mathfrak{g}_{m}\right)$ admits the decomposition

$$
\mathcal{R}(\mathfrak{g})=\mathbb{R} \oplus E_{m} \oplus \mathcal{R}(\mathfrak{h}) \oplus \mathcal{P}(\mathfrak{h}) \oplus \odot^{2} E_{m}
$$

The space $\mathcal{P}(\mathfrak{h})$ does not contain any $\mathfrak{h}$-invariant one-dimensional subspace [12], hence $P_{m}=0$. For $X, Y, Z \in E_{m}$ it holds

$$
\begin{aligned}
\mu\left(p_{m} \wedge Z\right) R_{0 m}(X, Y)=\mu\left(p_{m} \wedge Z\right) R_{m}(X, Y) & =\left(\left(p_{m} \wedge Z\right) \cdot R_{m}\right)(X, Y) \\
& =\left[p_{m} \wedge Z, R_{0 m}(X, Y)\right]=-p_{m} \wedge R_{0 m}(X, Y) Z .
\end{aligned}
$$

This implies $R_{0 m}=0$. Thus over the current coordinate neighborhood it holds $R_{0}=0$ and $P=0$. The same can be shown for the other possible holonomy algebras. We get $R\left(p^{\perp}, p^{\perp}\right)=0$. In [18] it is proved that in this case the coordinates can be chosen in such a way that

$$
g=2 \mathrm{~d} v \mathrm{~d} u+\sum_{i=1}^{n}\left(\mathrm{~d} x^{i}\right)^{2}+H(\mathrm{~d} u)^{2} .
$$

In particular, $\mathfrak{h}=0$ and either $\mathfrak{g}_{m}=p_{m} \wedge E_{m}$, or $\mathfrak{g}_{m}=\mathbb{R} p_{m} \wedge q_{m}+p_{m} \wedge E_{m}$. Consider these two cases.

Case 1. Suppose that $\mathfrak{g}_{m}=p_{m} \wedge E_{m}$. Then $\partial_{v} H=0$. In [2] it is shown that the covariant curvature tensor and its covariant derivative have the form

$$
\begin{aligned}
R & =\frac{1}{2}\left(\partial_{i} \partial_{j} H\right)\left(q^{\prime} \wedge e^{i} \vee q^{\prime} \wedge e^{j}\right), \\
\nabla R & =\frac{1}{2}\left(\partial_{k} \partial_{i} \partial_{j} H\right) e^{k} \otimes\left(q^{\prime} \wedge e^{i} \vee q^{\prime} \wedge e^{j}\right)+\frac{1}{2}\left(\partial_{u} \partial_{i} \partial_{j} H\right) q^{\prime} \otimes\left(q^{\prime} \wedge e^{i} \vee q^{\prime} \wedge e^{j}\right),
\end{aligned}
$$

where $e^{i}=\mathrm{d} x^{i}$ and $q^{\prime}=\mathrm{d} u$. The condition (1) is equivalent to

$$
\partial_{k} \partial_{i} \partial_{j} H=\theta_{k} \partial_{i} \partial_{j} H, \quad \partial_{u} \partial_{i} \partial_{j} H=\theta_{u} \partial_{i} \partial_{j} H
$$

where $\theta_{k}=\theta\left(\partial_{k}\right)$ and $\theta_{u}=\theta\left(\partial_{u}\right)$. If $\partial_{i} \partial_{j} H \neq 0$ for some $i, j$ on some open subspace, then

$$
\theta_{k}=\partial_{k} \ln \left|\partial_{i} \partial_{j} H\right|, \quad \theta_{u}=\partial_{u} \ln \left|\partial_{i} \partial_{j} H\right|,
$$

i.e. $\mathrm{d} \theta=0$ and there exists a function $f$ such that $\theta=\mathrm{d} f$. We get

$$
\partial_{k}\left(\ln \left|\partial_{i} \partial_{j} H\right|-f\right)=\partial_{u}\left(\ln \left|\partial_{i} \partial_{j} H\right|-f\right)=0,
$$

i.e.

$$
\ln \left|\partial_{i} \partial_{j} H\right|=f+c_{i j}, \quad c_{i j} \in \mathbb{R}, \quad c_{i j}=c_{j i} .
$$

Thus,

$$
\partial_{i} \partial_{j} H=e^{f} C_{i j}, \quad C_{i j}=e^{c_{i j}}
$$


Consider the new coordinates

$$
\tilde{v}=v, \quad \tilde{x}^{i}=a_{j}^{i} x^{j}, \quad \tilde{u}=u,
$$

where $a_{j}^{i}$ is an orthogonal matrix. With respect to these coordinates the metric $g$ takes the same form and it holds

$$
\tilde{\partial}_{i} \tilde{\partial}_{j} \tilde{H}=e^{\tilde{f}} a_{i}^{r} a_{j}^{s} C_{r s}
$$

The orthogonal transformation $a_{i}^{j}$ can be chosen in such a way that the matrix $\tilde{C}_{i j}=a_{i}^{r} a_{j}^{s} C_{r s}$ is diagonal with the diagonal elements $\lambda_{1}, \ldots, \lambda_{n}$. Assume that $\left|\lambda_{1}\right| \geq \cdots \geq\left|\lambda_{n}\right|$. Thus it holds

$$
\partial_{i} \partial_{j} H=e^{f} \delta_{i j} \lambda_{i}, \quad \lambda_{i} \in \mathbb{R} .
$$

If $\lambda_{2}=\cdots=\lambda_{n}=0$, then

$$
H=F\left(x^{1}, u\right)+\sum_{i=2}^{n} G_{i}(u) x^{i} .
$$

Consider the new coordinates given by the inverse transformation

$$
u=\tilde{u}, \quad x^{i}=\tilde{x}^{i}+b^{i}(\tilde{u}), \quad v=\tilde{v}-\sum_{j} \frac{\mathrm{d} b^{j}(\tilde{u})}{\mathrm{d} \tilde{u}} \tilde{x}^{j}
$$

such that $2 \frac{\mathrm{d}^{2} b^{j}(u)}{(\mathrm{d} u)^{2}}=G_{j}(u)$ and $b^{1}(u)=0$. With respect to the new coordinates it holds $H=F\left(x^{1}, u\right)$ and we obtain the Case I of the formulation of the theorem.

Suppose that $\lambda_{2} \neq 0$. From the above we get that if $i \neq j$, then $\partial_{i} \partial_{j} H=0$, i.e. $H$ is of the form $H=\sum_{i} H_{i}\left(x^{i}\right)$, and $\frac{d^{2} H_{i}}{\left(d x^{i}\right)^{2}}=e^{f} \lambda_{i}$. Taking $i=1,2$ and differentiating the last equality with respect to $\partial_{j}$, we get $\partial_{j} f=0$, i.e. $f$ depends only on $u$. Now it is clear that

$$
H=\frac{1}{2} e^{f(u)} \lambda_{i}\left(x^{i}\right)^{2}+B_{i}(u) x^{i}+K(u) .
$$

Let $F(u)=\frac{1}{2} e^{f(u)}$. From the results of [2] it follows that the coordinates can be chosen in such a way that $H=F(u) \lambda_{i}\left(x^{i}\right)^{2}$.

Case 2. Suppose that $\mathfrak{g}_{m}=\mathbb{R} p_{m} \wedge q_{m}+p_{m} \wedge E_{m}$. The curvature tensor $R_{m}$ is given by the elements $\lambda_{m}, \vec{v}_{m}$ and $T_{m}$. It holds

$$
\begin{aligned}
\mu\left(p_{m} \wedge q_{m}\right)\left(-\lambda_{m} p_{m} \wedge q_{m}-p_{m}\right. & \left.\wedge \vec{v}_{m}\right) \\
= & \left(\left(p_{m} \wedge q_{m}\right) \cdot R_{m}\right)\left(p_{m}, q_{m}\right)=\left[p_{m} \wedge q_{m}, R\left(p_{m}, q_{m}\right)\right]=p_{m} \wedge \vec{v}_{m}
\end{aligned}
$$

hence

$$
\mu\left(p_{m} \wedge q_{m}\right) \lambda_{m}=0, \quad\left(\mu\left(p_{m} \wedge q_{m}\right)+1\right) \vec{v}_{m}=0 .
$$

Similarly, $\left(\left(p_{m} \wedge q_{m}\right) \cdot R_{m}\right)\left(X, q_{m}\right)=\mu\left(p_{m} \wedge q_{m}\right) R_{m}\left(X, q_{m}\right)$, for $X \in E_{m}$, implies

$$
\mu\left(p_{m} \wedge q_{m}\right) \vec{v}_{m}=0, \quad\left(\mu\left(p_{m} \wedge q_{m}\right)+1\right) T_{m}=0 .
$$

In the same way, using an element $p_{m} \wedge X \in \mathfrak{g}_{m}$, we get

$$
\begin{aligned}
\mu\left(p_{m} \wedge X\right) \lambda_{m}=0, \quad \mu\left(p_{m} \wedge X\right) \vec{v}_{m}= & \lambda_{m} X, \\
& \mu\left(p_{m} \wedge X\right) \vec{v}_{m}=0, \quad g\left(\vec{v}_{m}, Y\right) X=\mu\left(p_{m} \wedge X\right) T_{m}(Y) .
\end{aligned}
$$

The obtained equalities imply $\vec{v}_{m}=0$ and $\lambda_{m}=0$. Consequently, over this coordinate neighborhood, $\lambda=0$ and $\vec{v}=0$. This shows that this coordinate neighborhood is the same as in Case 1. 


\subsection{The case of analytic $(M, g)$}

Suppose that $(M, g)$ is analytic. In this case, Theorem 1 can be reformulated in the following way:

Theorem 2 Let $(M, g)$ be an analytic Lorentzian manifold of dimension $n+2 \geq 3$. Then $(M, g)$ is recurrent and not locally symmetric if and only if one of the following holds:

I. In a neighborhood of each point of $M$ there exist coordinates $v, x^{1}, \ldots, x^{n}, u$ and a function $H\left(x^{1}, u\right)$ such that

$$
g=2 d v d u+\sum_{i=1}^{n}\left(d x^{i}\right)^{2}+H\left(x^{1}, u\right)(d u)^{2},
$$

and $\partial_{1}^{2} H$ is not constant for some system of coordinates.

In this case if $n \geq 2$, then the manifold is locally a product of the three-dimensional recurrent Lorentzian manifold with the coordinates $v, x^{1}, u$ and of the flat Riemannian manifold with the coordinates $x^{2}, \ldots, x^{n}$.

II. There exist real numbers $\lambda_{1}, \ldots, \lambda_{n}$ with $\left|\lambda_{1}\right| \geq \cdots \geq\left|\lambda_{n}\right|, \lambda_{2} \neq 0$, and an analytic function $F: U \subset \mathbb{R} \rightarrow \mathbb{R}$ with $\frac{d F}{d u} \neq 0$, and in a neighborhood of each point of $M$ there exist coordinates $v, x^{1}, \ldots, x^{n}, u$ such that

$$
g=2 d v d u+\sum_{i=1}^{n}\left(d x^{i}\right)^{2}+F(u) \lambda_{i}\left(x^{i}\right)^{2}(d u)^{2} .
$$

The manifold $(M, g)$ is locally indecomposable if and only if all $\lambda_{i}$ are non-zero.

If for some $r(2 \leq r<n)$ it holds $\lambda_{r} \neq 0$ and $\lambda_{r+1}=\cdots=\lambda_{n}=0$, then $(M, g)$ is locally a product of the recurrent Lorentzian manifold with the coordinates $v, x^{1}, \ldots, x^{r}, u$ and of the flat Riemannian manifold with the coordinates $x^{r+1}, \ldots, x^{n}$.

In particular, the theorem states that in the second case the metric is the same in each coordinate neighborhood.

Proof. Suppose that a point $m$ belongs to two coordinate neighborhoods with the coordinates $v, x^{1}, \ldots, x^{n}, u$ and $\tilde{v}, \tilde{x}^{1}, \ldots, \tilde{x}^{n}, \tilde{u}$. Suppose that for the first system of coordinates it holds $H=F(u) \lambda_{i}\left(x^{i}\right)^{2}, \lambda_{1}, \lambda_{2} \neq 0$, and $\frac{\mathrm{d} F}{\mathrm{~d} u} \neq 0$, i.e. the metric restricted to the first coordinate neighborhood is not flat. If in the second coordinate system the metric is flat, then on the intersection of the coordinate domains it holds $\frac{\mathrm{d} F}{\mathrm{~d} u}=0$. Since $F$ is analytic, this implies $\frac{\mathrm{d} F}{\mathrm{~d} u}=0$ for all points of the first coordinate neighborhood and we get a contradiction (this is the only place, where we use the analyticity). Since the metric restricted to the second coordinate neighborhood is not flat, the parallel vector field $\tilde{\partial}_{v}$ is defined up to a constant and we may assume that $\tilde{\partial}_{v}=\partial_{v}$. Then the transformation of coordinates must have the form

$$
u=\tilde{u}+c, \quad x^{i}=a_{j}^{i} \tilde{x}^{j}+b^{i}(\tilde{u}), \quad v=\tilde{v}-\sum_{j} a_{i}^{j} \frac{\mathrm{d} b^{j}(\tilde{u})}{\mathrm{d} \tilde{u}} \tilde{x}^{i}+d(\tilde{u}) .
$$

where $c \in \mathbb{R}, a_{i}^{j}$ is an orthogonal matrix, and $b^{i}(\tilde{u}), d(\tilde{u})$ are some functions of $\tilde{u}[2]$. Clearly, the metric written in the second coordinate system can not be as in Case I of Theorem 1, i.e. it holds

$$
\tilde{H}=\tilde{F}(\tilde{u}) \tilde{\lambda}_{i}\left(\tilde{x}^{i}\right)^{2}
$$


Note that

$$
\tilde{F}(\tilde{u}) \delta_{i j} \tilde{\lambda}_{i}=F(\tilde{u}+c) \delta_{k l} \lambda_{k} a_{i}^{k} a_{j}^{l} .
$$

Since the matrix $a_{i}^{j}$ is orthogonal, after some change

$$
\left(F(\tilde{u}), \tilde{\lambda}_{i}\right) \mapsto\left(\frac{1}{C} F(\tilde{u}), C \tilde{\lambda}_{i}\right), \quad C \neq 0,
$$

we obtain $\tilde{\lambda}_{i}=\lambda_{i}$ and $\tilde{F}(\tilde{u})=F(\tilde{u}+c)$. After the transformation $\tilde{u} \mapsto \tilde{u}-c$, we get $\tilde{F}=F$. This proves the theorem.

\section{$7 \quad$ Lorentzian symmetric spaces}

Classification of simply connected Riemannian symmetric spaces is a classical result of Élie Cartan [4]. Simply connected Lorentzian symmetric spaces are classified by Cahen and Wallach $[6,7]$. Here we show how the last result can be reproved using the holonomy theory. It is wellknown that a simply connected pseudo-Riemannian symmetric space is uniquely defined by the pair $(\mathfrak{g}, R)$, where $\mathfrak{g}$ is its holonomy algebra and $R$ is its curvature tensor at a fix point. Such pair satisfies $R \in \mathcal{R}(\mathfrak{g}), \mathfrak{g}$ annihilates $R$ and the image of $R$ coincides with $\mathfrak{g}$. Note that $R$ can be defined up to a positive constant. Now we describe such pairs for $\mathfrak{g} \subset \mathfrak{s o}(1, n+1)$. It is enough to consider indecomposable spaces, i.e. we may assume that $\mathfrak{g} \subset \mathfrak{s o}(1, n+1)$ is weakly irreducible. If $\mathfrak{g}=\mathfrak{s o}(1, n+1)$, then such $R$ constitute a one-dimensional space without the zero. The connected components of this space define de Sitter and Anti de Sitter spaces. These are the only indecomposable simply connected Lorentzian symmetric spaces with semisimple isometry group (equivalently, with reductive holonomy algebra). Now we suppose that $\mathfrak{g} \subset \mathfrak{s i m}(n)$. In the same way as we did in Section 6 , we conclude that $\mathfrak{g}=p \wedge E$ and $R$ is given by a symmetric endomorphism $T$ of $E$ (in notation of Section 4). Thus such pair corresponds to a pp-wave (4). Equation (9) shows that $\partial_{k} \partial_{i} \partial_{j} H=\partial_{u} \partial_{i} \partial_{j} H=0$, i.e. $H=a_{i j} x^{i} x^{j}+b_{i}(u) x^{i}+c(u)$. Changing the coordinates, we get $H=\sum_{i} \lambda_{i}\left(x^{i}\right)^{2}, \lambda_{i} \in \mathbb{R}$. Metric (4) with such $H$ is defined on $\mathbb{R}^{n+2}$ and it is complete. These symmetric spaces are called the Cahen-Wallach spaces.

\section{The Weyl conformal curvature tensor of a Walker met- ric}

Below we will need the expression for the Weyl tensor $W$ of a Walker metric in terms of notations of Section 4. This expression is obtained in [13]. It holds,

$$
W=R+R_{L}
$$


where $R_{L}$ is defined as follows:

$$
\begin{aligned}
R_{L}(p, X) & =\frac{1}{n} p \wedge\left(\operatorname{Ric}(h)+\frac{(n-1) \lambda-s_{0}}{n+1} \mathrm{id}\right) X \\
R_{L}(p, q) & =\frac{1}{n}\left(\frac{2 n \lambda-s_{0}}{n+1} p \wedge q+p \wedge(\vec{v}-\widetilde{\operatorname{Ric} P})\right) \\
R_{L}(X, Y) & =\frac{1}{n}(p \wedge((X \wedge Y)(\vec{v}-\widetilde{\operatorname{Ric}} P)) \\
& \left.+\left(\operatorname{Ric}(h)-\frac{s}{2(n+1)}\right) X \wedge Y+X \wedge\left(\operatorname{Ric}(h)-\frac{s}{2(n+1)}\right) Y\right) \\
R_{L}(X, q) & =\frac{1}{n}((\operatorname{tr} T) p \wedge X+g(X, \vec{v}-\widetilde{\operatorname{Ric}} P) p \wedge q+X \wedge(\vec{v}-\widetilde{\operatorname{Ric} P}) \\
& \left.+\left(\operatorname{Ric}(h)+\frac{(n-1) \lambda-s_{0}}{n+1} \mathrm{id}\right) X \wedge q\right)
\end{aligned}
$$

where $\operatorname{Ric}(h)$ is the Ricci operator of the metric $h, s=2 \lambda+s_{0}$ is the scalar curvature of $g$ and $S_{0}$ is the scalar curvature of $h$. This expression is used in [13] to find all conformally flat Walker metrics.

\section{$9 \quad$ Lorentzian manifolds with recurrent and parallel Weyl tensor}

Conformally symmetric Lorentzian manifolds, i.e. Lorentzian manifolds with parallel Weyl tensor $W$ are classified by Derdzinski and Roter $[8,9]$. These spaces are exhausted by conformally flat spaces, i.e. with $W=0$, by locally symmetric spaces, i.e. with $\nabla R=0$, and by some special pp-waves. As a generalization of this condition one consider conformally recurrent spaces, i.e. with recurrent Weyl tensor, $\nabla W=\theta \otimes W$, see e.g. [19, 23]. We prove the following theorem.

Theorem 3 Let $(M, g)$ be a locally indecomposable Lorentzian manifold of dimension $n+2 \geq 4$ with a recurrent Weyl tensor $W$, then either $W=0$, or $\nabla R=0$, or locally $g$ has the form

$$
g=2 d v 2 d u+\sum_{i=1}^{n}\left(d x^{i}\right)^{2}+\left(a(u) \sum_{i=1}^{n}\left(x^{i}\right)^{2}+F(u) \sum_{i=1}^{n} \lambda_{i}\left(x^{i}\right)^{2}\right)(d u)^{2},
$$

where $a(u), F(u)$ are functions, and $\lambda_{i} \in \mathbb{R}, \sum_{i=1}^{n} \lambda_{i}=0$.

Note that the for the above metric it holds $\nabla W=0$ if and only if $\frac{\mathrm{d} F(u)}{\mathrm{d} u}=0$. In particular, we recover the result by Derdzinski and Roter. Next, $\nabla R=0$ if and only if $\frac{\mathrm{d} a(u)}{\mathrm{d} u}=\frac{\mathrm{d} F(u)}{\mathrm{d} u}=0$. Also, $\nabla R=\theta \otimes R$ if and only if $a(u)=F(u)$, or $a(u)=0$, or all $\lambda_{i}=0$. Finally, $W=0$ if and only if all $\lambda_{i}=0$ or $F(u)=0[13]$.

Proof of Theorem 3. The proof is very similar to the proof of Theorem 1 and we omit some obvious computations. Suppose that $W \neq 0$ and $\nabla R \neq 0$. Let $\mathfrak{g} \subset \mathfrak{s o}(1, n+1)$ be the holonomy algebra of $(M, g)$ at a point $m \in M$. Then $\mathfrak{g}$ preserves the line in the space $\mathcal{R}(\mathfrak{g})$ spanned by $W_{m}$. For $\mathfrak{g}=\mathfrak{s o}(1, n+1)$ this would imply $W_{m}=0$, which follows from [1]. Hence, $\mathfrak{g} \subset \mathfrak{s i m}(n)$. Suppose that $W_{m} \neq 0$. 
Lemma 2 The manifold $(M, g)$ is a pp-wave, i.e. $\mathfrak{g}=p_{m} \wedge E_{m}$.

Proof. For each $\xi \in \mathfrak{g}$ it holds $\xi \cdot W_{m}=\mu(\xi) W_{m}$, where $\mu: \mathfrak{g} \rightarrow \mathbb{R}$ is a linear map.

First suppose that $\mathfrak{h}=0$. Then either $\mathfrak{g}=\mathbb{R} p_{m} \wedge q_{m}+p_{m} \wedge E_{m}$, or $\mathfrak{g}=p_{m} \wedge E_{m}$. Suppose that $\mathfrak{g}=\mathbb{R} p_{m} \wedge q_{m}+p_{m} \wedge E_{m}$. Then we may assume that $\lambda_{m} \neq 0$ or $\vec{v}_{m} \neq 0$. Let $Z \in E_{m}$ be not proportional to $\vec{v}_{m}$. Considering $\left(\left(p_{m} \wedge Z\right) \cdot W_{m}\right)\left(p_{m}, q_{m}\right)$, we obtain $\lambda_{m}=0$. Considering $\left(\left(p_{m} \wedge Z\right) \cdot W_{m}\right)\left(Z_{m}, q_{m}\right)$, we get $\vec{v}_{m}=0$. We conclude that $\mathfrak{g}=p_{m} \wedge E_{m}$.

Suppose now that $\mathfrak{h} \neq 0$. Let $A \in \mathfrak{h}$ and let $\xi$ be either $A$, or $A+\varphi(A) p_{m} \wedge q_{m}$, or $A+p_{m} \wedge$ $\psi(A)$ depending on the type of $\mathfrak{g}$. Note that any 1-dimensional representation of $\mathfrak{h}$ is trivial, consequently, $\mu(\xi)=0$. Using this and considering the projection of $\left(\xi \cdot W_{m}\right)(X, Y)$ to $\mathfrak{s o}(n)$, where $X, Y \in E_{m}$, we get

$$
A \cdot\left(\frac{1}{n}\left(\cdot \wedge\left(\vec{v}_{m}-\widetilde{\operatorname{Ric}} P_{m}\right)\right)+P_{m}\right)=0,
$$

where we consider the representation of $\mathfrak{h}$ in the space $\mathcal{P}(\mathfrak{s o}(n))$. The module $\mathcal{P}(\mathfrak{h})$ never contains non-zero elements annihilated by $\mathfrak{h}[12]$. If $P_{m}=0$, then from the above equality it follows that $\vec{v}_{m}=0$. Otherwise, since $\mathfrak{h} \neq 0$, there exists $A \in \mathfrak{h}$ such that $A \cdot P_{m} \neq 0$. This implies that $0 \neq \wedge A\left(\vec{v}_{m}-\widetilde{\operatorname{Ric}} P_{m}\right) \in \mathcal{P}(\mathfrak{h})$. Consequently, $\mathfrak{h}=\mathfrak{s o}(n)$. We conclude that

$$
P_{m}=\frac{1}{n}\left(\cdot \wedge\left(\vec{v}_{m}-\widetilde{\operatorname{Ric}} P_{m}\right)\right) .
$$

Applying $\widetilde{\text { Ric }}$, we get $\widetilde{\operatorname{Ric}} P_{m}=(1-n) \vec{v}_{m}$, and we conclude that

$$
P_{m}=-\cdot \wedge \vec{v}_{m} .
$$

This shows that the expression of $W_{m}$ does not include $\vec{v}_{m}$ and $P_{m}$. Considering $\left(\left(p_{m} \wedge Z\right)\right.$. $\left.W_{m}\right)\left(p_{m}, q_{m}\right), Z \in E_{m}$, we get that $\operatorname{Ric}(h)_{m}=c_{1} \operatorname{id}_{E_{m}}$, where $c_{1}$ can be expressed in terms of $\lambda_{m}$ and $s_{0 m}$. Taking the trace, we get a relation between $\lambda_{m}$ and $s_{0 m}$. Taking $\xi$ as above, using the equality $\operatorname{pr}_{\mathfrak{s o}(n)}((\xi \cdot W)(X, Y))=0, X, Y \in E_{m}$, and expressing $R(h)_{m}$ in terms of the Weyl tensor of $h, \operatorname{Ric}(h)_{m}$ and $s_{0 m}$, we get another relation between $\lambda_{m}$ and $s_{0 m}$. Then we conclude that $\lambda_{m}=s_{0 m}=0, \operatorname{Ric}(h)_{m}=0$, and $R(h)_{m}=0$. Now $W_{m}$ depends only on $T_{m}$. Since $\mathfrak{h} \neq 0$ and $R(h)=0$, we may assume that $P_{m} \neq 0$, and we have just seen that this implies $\mathfrak{h}=\mathfrak{s o}(n)$. Taking $A \in \mathfrak{s o}(n)$ and considering $\left(A \cdot W_{m}\right)\left(X, q_{m}\right)$, we get $T_{m} A=A T_{m}$. The Schur Lemma implies that $T_{m}$ is proportional to $\operatorname{id}_{E_{m}}$. Thus, $W_{m}=0$. And we get a contradiction. Thus, $\mathfrak{h}=0$ and $\mathfrak{g}=p_{m} \wedge E_{m}$, this proves the lemma.

Now we should find all functions $H$ such the Weyl tensor of metric (4) of a pp-wave is recurrent, i.e. $\nabla W=\theta \otimes W$ for a 1 -form $\theta$. For $W$ and $\nabla W$ we get the formulas (8) and (9) with $\partial_{i} \partial_{j} H$ replaced by $\partial_{i} \partial_{j} H-\frac{1}{n} \delta_{i j} \Delta H$, where $\Delta=\sum_{k=1}^{n} \partial_{k}^{2}$. We obtain the equations

$$
\begin{aligned}
& \partial_{k}\left(\partial_{i} \partial_{j} H-\frac{1}{n} \delta_{i j} \Delta H\right)=\theta_{k}\left(\partial_{i} \partial_{j} H-\frac{1}{n} \delta_{i j} \Delta H\right), \\
& \partial_{u}\left(\partial_{i} \partial_{j} H-\frac{1}{n} \delta_{i j} \Delta H\right)=\theta_{u}\left(\partial_{i} \partial_{j} H-\frac{1}{n} \delta_{i j} \Delta H\right),
\end{aligned}
$$

where $\theta_{k}=\theta\left(\partial_{k}\right)$ and $\theta_{u}=\theta\left(\partial_{u}\right)$. As in Section 6 , we get

$$
\partial_{i} \partial_{j} H-\frac{1}{n} \delta_{i j} \Delta H=e^{f} C_{i j}, \quad C_{i j}=C_{j i} \in \mathbb{R}
$$


for all $i, j$. Since $\Delta$ is invariant with respect to an orthogonal transformation of the coordinates $x^{1}, \ldots, x^{n}$, we may apply a transformation as in Section 6 , ad we may assume that

$$
\partial_{i} \partial_{j} H-\frac{1}{n} \delta_{i j} \Delta H=e^{f} \delta_{i j} \lambda_{i}, \quad \lambda_{i} \in \mathbb{R}
$$

If $i \neq j$, then $\partial_{i} \partial_{j} H=0$, i.e. $H$ is of the form $H=\sum_{i=1}^{n} H_{i}\left(x^{i}, u\right)$. We get the system of equations

$$
\partial_{i}^{2} H_{i}-\frac{1}{n} \sum_{k=1}^{n} \partial_{k}^{2} H_{k}=e^{f} \lambda_{i} .
$$

We may view this system as a system of linear equations with respect to the unknowns $\partial_{i}^{2} H_{i}$. The rank of this system is equal to $n-1$. Summarizing the equations, we see that if a solution exists, then $\sum_{i=1}^{n} \lambda_{i}=0$. In this case the dimension of solutions equals 1 , and we have $\partial_{i}^{2} H_{i}=\frac{1}{2} a+e^{f} \lambda_{i}$ for a function $a$. This implies that both $a$ and $f$ are functions depending only on $u$. We obtain

$$
H=a(u) \sum_{i=1}^{n}\left(x^{i}\right)^{2}+F(u) \lambda_{i}\left(x^{i}\right)^{2},
$$

where $F(u)=\frac{1}{2} e^{f(u)}$ and we assume that the terms linear in $x^{i}$ are zero, since we can get read of them using a transformation of coordinates. The theorem is proved.

Results about four-dimensional conformally recurrent Lorentzian spaces are collected in [21, Ch. 35].

\section{Two-symmetric Lorentzian manifolds}

In this section we consider two-symmetric Lorentzian manifolds, i.e. manifolds satisfying (2). The following theorem is proved in $[2]$.

Theorem 4 Let $(M, g)$ be a locally indecomposable Lorentzian manifold of dimension $n+2$. Then $(M, g)$ is two-symmetric if and only if locally there exist coordinates $v, x^{1}, \ldots, x^{n}, u$ such that

$$
g=2 d v d u+\sum_{i=1}^{n}\left(d x^{i}\right)^{2}+\left(H_{i j} u+F_{i j}\right) x^{i} x^{j}(d u)^{2},
$$

where $H_{i j}$ is a nonzero diagonal real matrix with the diagonal elements $\lambda_{1} \leq \cdots \leq \lambda_{n}$, and $F_{i j}$ is a symmetric real matrix.

Detailed investigation of two-symmetric Lorentzian spaces initiated Senovilla in [20], where it is proven that any two-symmetric Lorentzian space admits a parallel isotropic vector field, i.e. locally the metric has the form (3) with $\partial_{v} H=0$.

Now we explain the proof of the above theorem from [2]. The assumption that a Lorentzian manifold $(M, g)$ is two-symmetric implies that the holonomy algebra $\mathfrak{g} \subset \mathfrak{s o}(1, n+1)$ of $(M, g)$ at a point $m \in M$ annihilates the tensor $\nabla R_{m} \neq 0$. The tensor $\nabla R_{m}$ belongs to the $\mathfrak{g}$-module $\nabla \mathcal{R}(\mathfrak{g})$ that consists of linear maps from $\mathbb{R}^{1, n+1}$ to $\mathcal{R}(\mathfrak{g})$ satisfying the second Bianchi identity. The results from [22] show that the space $\nabla \mathcal{R}(\mathfrak{s o}(1, n+1))$ does not contain any non-zero element annihilated by $\mathfrak{s o}(1, n+1)$. Hence, $\mathfrak{g}$ can not coincide with $\mathfrak{s o}(1, n+1)$, and $\mathfrak{g}$ must be contained in $\mathfrak{s i m}(n)$. We show that the holonomy algebras $\mathfrak{g}$ of types I and III do not annihilate 
any non-zero element in $\nabla \mathcal{R}(\mathfrak{g})$, i.e. $\mathfrak{g}$ must be of type II or IV. In this case $(M, g)$ admits a parallel isotropic vector field, i.e. we reprove the result of [20]. Next, we prove a reduction lemma that allows to consider the following two cases: $\mathfrak{g}=\mathfrak{h}+p \wedge E$, where $\mathfrak{h} \subset \mathfrak{s o}(n)$ is an irreducible subalgebra, and $\mathfrak{g}=p \wedge E$.

We prove that the first case is impossible. For this we find the form of all tensors in $\nabla \mathcal{R}(\mathfrak{g})$ annihilated by $\mathfrak{g}$, it turns out that this space is one-dimensional. Then we may find the form of $\nabla R$. We calculate $\nabla$ Ric, and show that the Weyl conformal tensor $W$ is parallel $(\nabla W=0)$. Then, using the results of Derdzinski and Roter [8, 9] and of [13], we get a contradiction.

The second case corresponds to pp-waves (4). The condition $\nabla^{2} R=0$ and simple computations allow us to find the coordinate form of the metric.

The proof of this result from [2] especially shows the power of the methods introduced in this paper, since lately there appeared another more technical proof [5] that uses computations in local coordinates for metric (3).

\section{Recurrent symmetric bilinear forms}

In [3] Aminova proved that if an indecomposable Lorentzian manifold $(M, g)$ admits a parallel symmetric bilinear form not proportional to the metric, then the manifold admits a parallel isotropic vector field $p$, and the space of parallel symmetric bilinear forms is spanned over $\mathbb{R}$ by the metric $g$ and by $\tau \otimes \tau$, where $\tau=g(p, \cdot)$ is the dual 1-form to $p$. We generalize this result to the case of recurrent symmetric bilinear forms.

Theorem 5 If an indecomposable Lorentzian manifold $(M, g)$ admits a recurrent symmetric bilinear form not proportional to the metric, then the manifold has holonomy algebras contained in $\mathfrak{s i m}(n)$, in particular locally it is given by the metric (3) and locally it admits recurrent isotropic vector fields.

Let $g$ be given by (3) and suppose that it is indecomposable. If $\partial_{v}^{2} H=\partial_{i} \partial_{v} H=0$, then the coordinates can be chosen in such a way that $\partial_{v} H=0$, in this case any recurrent symmetric bilinear form equals to $f(\alpha g+\beta \tau \otimes \tau)$, where $\alpha, \beta \in \mathbb{R}, \tau=d u=g\left(\partial_{v}, \cdot\right)$, and $f$ is a function; if $\partial_{v}^{2} H \neq 0$ or $\partial_{i} \partial_{v} H \neq 0$, then any recurrent symmetric bilinear is proportional either to $g$, or to $\tau \otimes \tau$.

For the proof it is enough to find for the holonomy algebra $\mathfrak{g} \subset \mathfrak{s i m}(n)$ at a point $m \in M$ all invariant 1 -dimensional subspaces in $\odot^{2}\left(\mathbb{R}^{1, n+1}\right)^{*}$ preserved by $\mathfrak{g}$. For algebras of type $\mathrm{I}$ and III these subspaces are $\mathbb{R} g_{m}$ and $\mathbb{R} \tau_{m} \otimes \tau_{m}$. For algebras of type II and IV these subspaces are 1-dimensional subspaces in $\mathbb{R} g_{m} \oplus \mathbb{R} \tau_{m} \otimes \tau_{m}$. The condition $\partial_{v}^{2} H=\partial_{i} \partial_{v} H=0$ holds only for the holonomy algebras of type II and IV.

The above theorem can be used for studying Lorentzian manifolds with recurrent Ricci tensors. We see that one deals with a Walker metric, and the equations will be very similar to the Einstein equation on the Walker metric, see [14].

\section{Acknowledgements}

I am thankful to D.V. Alekseevsky for useful discussions and to Andrzej Derdzinski for helpful correspondence. 


\section{References}

[1] Alekseevsky D.V., Riemannian manifolds with exceptional holonomy groups, Funksional Anal. i Prilozhen. 2 (1968) 1-10.

[2] Alekseevsky D.V., Galaev A.S., Two-symmetric Lorentzian manifolds, J. Geom. Phys. 61 (2011) 2331-2340.

[3] Aminova A.V., Pseudo-Riemannian manifolds with common geodesics, Russian Math. Surveys 48 (1993) 105-160.

[4] Besse A.L., Einstein manifolds, Springer-Verlag, Berlin-Heidelberg-New York, 1987.

[5] Blanco O.F., Sánchez M., Senovilla J.M.M., Structure of second-order symmetric Lorentzian manifolds, arXiv:1101.5503.

[6] Cahen M., Wallach N., Lorentzian symmetric spaces, Bull. Amer. Math. Soc. 76 (1970) $585-591$.

[7] Cahen M., Lorentzian symmetric spaces, Acad. Roy. Belg. Bull. Cl. Sci. 9 (1998) 325-330.

[8] Derdzinski A., Roter W., On conformally symmetric manifolds with metrics of indices 0 and 1, Tensor (N.S.) 31 (1977) 255-259.

[9] Derdzinski A., Roter W., The local structure of conformally symmetric manifolds, Bull. Belg. Math. Soc. Simon Stevin 16 (2009) 117-128.

[10] Galaev A.S., Leistner T., Holonomy groups of Lorentzian manifolds: classification, examples, and applications, Recent developments in pseudo-Riemannian geometry, 53-96, ESI Lect. Math. Phys., Eur. Math. Soc., Zürich, 2008.

[11] Galaev A.S., The spaces of curvature tensors for holonomy algebras of Lorentzian manifolds, Diff. Geom. and its Applications 22 (2005) 1-18.

[12] Galaev A.S., One component of the curvature tensor of a Lorentzian manifold, J. Geom. Phys. 60 (2010) 962-971.

[13] Galaev A.S., Conformally flat Lorentzian manifolds with special holonomy groups, Sbornik Mathematics 204 (2013) 1264-1284.

[14] Galaev A.S., Leistner T. On the local structure of Lorentzian Einstein manifolds with parallel distribution of null lines, Class. Quantum Grav. 27 (2010) 225003.

[15] Joyce D., Riemannian holonomy groups and calibrated geometry, Oxford University Press, 2007.

[16] Kaigorodov V.R., Structure of the curvature of space-time, Journal of Soviet Mathematics 28 (1985) 256-273.

[17] Leistner T., On the classification of Lorentzian holonomy groups, J. Differential Geom. 76 (2007) 423-484.

[18] Leistner T., Screen bundles of Lorentzian manifolds and some generalisations of pp-waves, J. Geom. Phys. 56 (2006) 2117-2134. 
[19] Olszak Z., On conformally recurrent manifolds, I: Special distributions, Zesz. Nauk. Politech. Sl., Mat.-Fiz. 68 (1993) 213-225.

[20] Senovilla J.M., Second-order symmetric Lorentzian manifolds. I. Characterization and general results, Classical Quantum Gravity 25 (2008) 245011, 25 pp.

[21] H. Stephani, D. Kramer, M. MacCallum, C. Hoenselaers, E. Herlt, Exact solutions to Einstein's field equations, Second edition, CUP, 2003.

[22] Strichartz R.S., Linear algebra of curvature tensors and their covariant derivatives, Canad. J. Math. 40 (1988) 1105-1143.

[23] Suh Y.J., Kwon J.H., Conformally recurrent semi-Riemannian manifolds, Rocky Mountain J. Math. 35 (2005) 285-307.

[24] Walker A.G., On Ruse's spaces of recurrent curvature, Proc. London Math. Soc. 52 (1950) 36-64. 